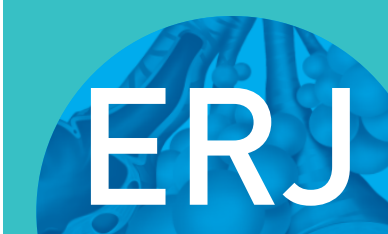

open research

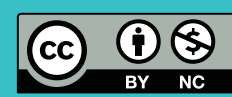

\section{The combination of inspiratory muscle training and high-flow nasal cannula oxygen therapy for promoting weaning outcomes in difficult-to-wean patients: protocol for a randomised controlled trial}

\author{
Irini Patsaki ${ }^{1}$, Anna Christakou ${ }^{1}$, Emmanouel Papadopoulos ${ }^{1}$, Martha Katartzi ${ }^{1}$, \\ Alexandros Kouvarakos ${ }^{1}$, Ilias Siempos ${ }^{2}$, Dimitris Tsimouris ${ }^{3}$, Anastasia Skoura ${ }^{4}$, \\ Antonina Xatzimina ${ }^{5}$, Sotirios Malachias ${ }^{2}$, Nikolaos Koulouris ${ }^{6}$, \\ Eirini Grammatopoulou ${ }^{5}$, Spiros Zakinthinos ${ }^{2}$ and Eleni Ischaki ${ }^{2}$
}

Affiliations: "Physiotherapy Dept, General Hospital of Athens "Evaggelismos", Athens, Greece. ${ }^{2} 1$ st Critical Care Dept, National and Kapodistrian University of Athens, General Hospital of Athens "Evaggelismos", Athens, Greece. ${ }^{3}$ Care and Rehabilitation Center "Kallisto", Athens, Greece. "Metropolitan Hospital of Athens, Athens, Greece. ${ }^{5}$ Dept of Physiotherapy, University of West Attica, Athens, Greece. ${ }^{6} 1$ st Respiratory Dept, National and Kapodistrian University of Athens, Athens, Greece.

Correspondence: Eleni Ischaki, 1st Critical Care Dept, National and Kapodistrian University of Athens, General Hospital of Athens “Evaggelismos”, Ipsilladou 45-47, 10676, Athens Greece. E-mail: eischakidayahoo.gr

\section{ABSTRACT}

Background: According to the literature, $20-30 \%$ of intubated patients are difficult to wean off mechanical ventilation and have a prolonged intensive care unit (ICU) stay with detrimental effects on muscle strength, functional ability and quality of life. Inspiratory muscle training (IMT) via a threshold device has been proposed as an effective exercise for minimising the effects of mechanical ventilation on respiratory muscles of critically ill patients with prolonged weaning. In addition, high-flow nasal cannula (HFNC) oxygen has been proved to provide efficient support for both high- and low-risk patients after extubation, thus preventing re-intubation.

Material and methods: A randomised controlled trial was designed to assess the efficacy of combining IMT and HFNC as therapeutic strategies for patients with high risk for weaning failure. Once patients with prognostic factors of difficult weaning are awake, ventilated with support settings and cooperative, they will be randomised to one of the two following study groups: intervention group (IMT and HFNC) and control group (IMT and Venturi mask). IMT will start as soon as possible. Each allocated oxygen delivery device will be applied immediately after extubation. IMT intervention will continue until patients' discharge from ICU. The primary outcome is the rate of weaning failure. Secondary outcomes are maximal inspiratory and expiratory strength, endurance of respiratory muscles, global muscle strength, functional ability and quality of life along with duration of ventilation (days) and ICU and hospital length of stay.

Conclusion: The present study could significantly contribute to knowledge of how best to treat patients with difficult weaning and high risk of re-intubation.

@ERSpublications

This RCT will provide a therapeutic strategic plan for difficult-to-wean patients, minimising their intubation period and length of ICU stay https://bit.ly/37CkxTB

Cite this article as: Patsaki I, Christakou A, Papadopoulos E, et al. The combination of inspiratory muscle training and high-flow nasal cannula oxygen therapy for promoting weaning outcomes in difficult-to-wean patients: protocol for a randomised controlled trial. ERJ Open Res 2020; 6: 000882020 [https://doi.org/10.1183/23120541.00088-2020].

This study is registered at www.clinicaltrials.gov with identifier number NCT03908658. Participants' data will be available following publication of the study.

Received: 24 Feb 2020 | Accepted after revision: 11 June 2020

Copyright $\odot$ ERS 2020. This article is open access and distributed under the terms of the Creative Commons Attribution Non-Commercial Licence 4.0. 


\section{Introduction}

Respiratory support by invasive mechanical ventilation is very common among critically ill patients in the intensive care unit (ICU). Weaning from mechanical ventilation after improvement of the underlying disease represents $40-50 \%$ of the total duration of mechanical ventilation and has a failure rate of about 26-42\% [1]. Weaning failure is a complex problem, attributed to different pathophysiological mechanisms such as respiratory disease, heart failure, obesity and respiratory muscle dysfunction, which in turn leads to prolonged mechanical ventilation [2,3]. In addition, the extension of mechanical ventilation promotes diaphragmatic weakness due to both atrophy and contractile dysfunction [4] and increases the risk of further complications, such as infections and critical illness neuromuscular syndromes with long-term consequences [5]. Weaning from mechanical ventilation should be considered as soon as possible to reduce its deleterious consequences. Nevertheless, weaning and extubation remains a challenging procedure, and when it is prolonged, re-intubation rates and in-hospital mortality increase by up to $32 \%[1]$.

Since 1980, both case reports and randomised controlled trials (RCTs) have suggested that inspiratory muscle training (IMT) in ventilator-dependent patients is associated with increased maximal inspiratory muscle strength (maximal inspiratory pressure (MIP)) [6-8] and weaning success [6-9]. In a recent meta-analysis [9] it is well documented that IMT is feasible and well tolerated in critically ill patients and that it could be an effective approach for patients that demonstrate diaphragmatic weakness. Vorona et al. [10] demonstrated that the increase in inspiratory muscle strength tended to be greater when using threshold loading devices compared to others. Threshold loading devices enable accurate control of muscle loading independent of inspiratory flow or patient respiratory mechanics. Further research is needed to determine the clinical significance of IMT.

After extubation, conventional oxygen delivery systems (i.e. nasal prongs, Venturi mask) with or without addition of noninvasive positive pressure ventilation are used to support the respiratory system and thus to avoid re-intubation [10]. Over the past decade, high-flow nasal cannula (HFNC) therapy has been introduced as an oxygen delivery device. It consists of an air/oxygen blender that can deliver a fraction of oxygen from $21 \%$ to $100 \%$ with a flow rate up to $60 \mathrm{~L} \cdot \mathrm{min}^{-1}$. The gas mixture is driven through a heated humidifier and a single limb heated inspiratory circuit to a wide-bore nasal cannula [11]. HFNC exerts its benefits through a variety of mechanisms such as delivery of constant inspiratory oxygen fraction $\left(\mathrm{F}_{\mathrm{IO}_{2}}\right)$ values, continued hydration of airways, generation of flow-related positive airway pressures and washout of $\mathrm{CO}_{2}$ from anatomical dead space $[11,12]$. Many studies support its use after extubation in ICU with promising results on rates of re-intubation, even among patients with high risk for re-intubation [13-15].

We hypothesise that the combined intervention of IMT and the application of HFNC immediately after extubation will: 1) decrease the rate of weaning failure; 2) increase the muscle strength and endurance, functional ability and quality of life at hospital discharge; and 3) decrease the ICU and hospital length of stay in patients at high risk for weaning failure, compared to IMT alone or HFNC alone.

The study has been approved by the Scientific Council and the Ethics Committee of "Evaggelismos" Hospital. The study has been registered in ClinicalTrials.gov (ClinicalTrials.gov ID: NCT03908658).

\section{Subjects and methods \\ Study subjects}

All patients from a general multidisciplinary ICU will be screened daily for prognostic factors for weaning failure. In addition, eligible patients must be alert and able to actively participate in the training (Riker Sedation Agitation Scale $\geqslant 4$ ) in order to be included in the study. The consent is taken from a patient's next of kin, after providing a full and detailed description of the study. Once written consent is given, the patient will be randomised (random concealed allocation) to receive either IMT and HFNC (intervention group) or IMT and Venturi mask (control group).

\section{Inclusion criteria}

Patients will be included if they are hospitalised in a multidisciplinary ICU and have one of the following:

1. age $>65$ years

2. body mass index $(\mathrm{BMI})>35 \mathrm{~kg} \cdot \mathrm{m}^{-2}$

3. weak cough

4. heart (i.e. heart failure) and lung comorbidities (i.e. chronic obstructive pulmonary disease)

5. hypercapnia during mechanical ventilation

6. $>72 \mathrm{~h}$ on mechanical ventilation

7. failed first spontaneous breathing trial 
Exclusion criteria

Patients will be excluded if they have:

1. Glasgow coma scale $<13$

2. pre-existing neuromuscular disease or deformity of spinal cord

3. terminal disease

4. haemodynamic instability requiring $>0.1 \mu \mathrm{g} \cdot \mathrm{kg}^{-1} \cdot \mathrm{min}^{-1}$ noradrenaline

5. immediate need of noninvasive ventilation after extubation

6. a pre-existing tracheostomy or are planned for tracheostomy

\section{Study design}

This is a prospective RCT. Randomisation for the study will be provided by sealed opaque envelopes, and the procedure will be conducted by a researcher not involved in the study or involved in the patient's care.

This RCT aims to answer the following questions:

1. Does IMT combined with HFNC decrease the weaning failure rate and the duration of mechanical ventilation in high risk for weaning failure patients?

2. Does the combined intervention of IMT and HFNC increase muscle strength and endurance, functional ability and quality of life at hospital discharge, and decrease hospital length of stay?

In our department, we use HFNC as a standard of care in patients with high risk for extubation failure, and our result will be compared with our standard of care.

Once a patient fulfils the inclusion criteria and does not have any exclusions, they will be randomised to either the intervention or the control group. Once they are awake, co-operative (Riker Sedation Agitation Scale $\geqslant 4$ ) and in a pressure support mode (pressure support ventilation $<15 \mathrm{cmH}_{2} \mathrm{O}$ and positive end-expiratory pressure $<10 \mathrm{cmH}_{2} \mathrm{O}$ ), patients in both groups will start inspiratory muscle training at $30 \%$ of MIP, as described below. Once extubated, patients in the intervention group will be put on HFNC, while patients in the control group will be fitted with a Venturi mask $\left(F_{\mathrm{IO}_{2}}\right.$ according to last known $F_{\mathrm{IO}_{2}}$ given while the patient was on a ventilator). The intervention of IMT will continue until ICU discharge.

The estimated sample size is 146 patients. The sample size estimation considered a two-sided significance level $(1-\alpha)$ of $95 \%$, a power of $80 \%$, equal numbers of patients in the intervention and control groups, a $20 \%$ absolute risk reduction from an estimated baseline weaning failure rate (i.e. re-intubation within $48 \mathrm{~h}$ after extubation) of $30 \%$, and a potential withdrawal or loss to follow-up rate of $15 \%$. The weaning failure rate of $30 \%$ for the control group (i.e. patients at high risk for re-intubation) was based on previous literature [16]. An interim analysis will be conducted when 73 patients have been enrolled to check for potential safety issues and to test for any difference between the compared groups in terms of the primary outcome (early efficacy).

\section{Interventions}

Inspiratory muscle training

IMT will be performed with the Threshold IMT inspiratory muscle trainer (Threshold IMT device HS730; Respironics, Murrysville, PA, USA), which has been used in patients with lung disease and in critically ill patients. This pressure threshold device is frequently used, and has been found to be very reliable as the patient has to generate a required flow-independent inspiratory pressure in order to open a valve in the device and start the training process [16]. The parameters used in the training protocol are based on previous studies of critically ill patients, which have shown that the intervention is safe and well tolerated by them $[7,9]$. Patients will commence with a load of $30 \%$ of $\mathrm{MIP}_{1 \mathrm{st}}$, which will be increased daily by $10 \%$ of $\mathrm{MIP}_{1 \mathrm{st}}$. They will perform five sets of 10 breaths (with 1 to $2 \mathrm{~min}$ of rest with ventilation support between each set), twice a day during week days and once during the weekend.

High-flow nasal cannula

Once intubated, patients in the intervention group will be transferred to HFNC. Initial settings will be: flow at $60 \mathrm{~L} \cdot \mathrm{min}^{-1}$ and $F_{\mathrm{IO}_{2}}$ based on the last set $F_{\mathrm{IO}_{2}}$ while the patient was still ventilated.

\section{Analysis}

The primary outcome is the rate of weaning failure, which is defined as re-intubation or application of noninvasive ventilation. Secondary outcomes are maximal inspiratory and expiratory strength, endurance of respiratory muscles, global muscle strength, functional ability and quality of life along with duration of ventilation (days) and ICU and hospital length of stay (table 1). 
Respiratory strength and fatigue measurement

MIP will be assessed with a unidirectional expiratory valve, which permits exhalation while inspiration is blocked, while maximal expiratory pressure is assessed with a unidirectional inspiratory valve, which permits inspiration while expiration is blocked, following the protocol used by Marini and colleagues for critically ill patients [17]. One side of the valve will be attached to the patient's endotracheal tube and the other side to a pressure manometer. Endurance of respiratory muscles will be calculated comparing the preloading and postloading values of the MIP through the calculation of fatigue resistance index [18].

\section{Muscle strength}

Muscle strength [19] will be assessed by summing the values obtained using the Medical Research Council (MRC) scale for muscle strength in six different muscle groups (shoulder abductors, forearm flexors, wrist flexors, hip flexors, knee extensors and plantar dorsiflexors) for both sides of the body. Each muscle group scores from 0 (no contraction) up to 5 (movement against gravity), and the total score ranges from 0 (worst outcome) to 60 (best outcome). The MRC scale for muscle strength has been found to have good reliability and validity [19].

\section{Functional ability and quality of life}

The functional independence measurement (FIM) will be used to assess functional ability. The FIM scale consists of 18 items (13 motor and five cognitive tasks) that assessed the level of independence. All items are rated on a seven-point ordinal scale that ranges from complete dependence to full independence. FIM has been found to have good reliability and validity [20].

Quality of life (QoL) will be assessed by the Short Form 36 Health Survey (SF-36) [21], which includes 36 items to measure eight QoL domains. More specifically, the following domains will be used from the SF-36: Physical Functioning, which includes 10 questions with possible scores from 1 to 3, Role Physical, which includes four yes/no questions, Social Functioning, which includes two questions with possible scores from 1 to 5 , and Mental Health, which includes nine questions with possible scores from 1 to 9 . A score of 1 is the worst outcome. Each domain is directly transformed into a $0-100$ scale on the assumption that each question carries equal weight. The higher the score, the less disability, i.e. a score of zero is equivalent to maximum disability and a score of 100 is equivalent to no disability.

In addition, the EuroQol 5D-5L [22] questionnaire will be used, which includes five domains and an optic analogue scale from 0 (worst) to 100 (best). Each domain (mobility, self-care, usual activities, pain/ discomfort, anxiety/depression) scores from 1 (best score) to 5 (worst score). The total score of all domains ranges from 5 (best score) to 25 (worst score). The total perceived quality of life will be graded by the patient.

All analyses will be conducted on an intention to treat basis using the non-parametric Mann-Whitney $U$ test for continuous variables and the Chi-squared test for categorical variables. A two-tailed p-value of $<0.05$ will be considered statistically significant. Any additional reviews of the data may be performed at the discretion of the Independent Data and Safety Monitoring Committee.

\section{TABLE 1 Outcome measures used in the study and their assessment time frame}

Outcomes - assessment tools

\begin{tabular}{l} 
\\
\hline MIP \\
MEP \\
Fatigue Resistance Index \\
Duration of mechanical \\
ventilation \\
Duration of ICU length of stay \\
MRC scale for muscle strength \\
Functional independence \\
measurement \\
SF-36
\end{tabular}

EuroQol 5D-5L
Time frame of assessments

$\begin{array}{ccccc}\begin{array}{c}\text { Baseline (pre-ICU } \\ \text { admission) }\end{array} & \begin{array}{c}\text { At } \\ \text { randomisation }\end{array} & \begin{array}{c}24 \mathrm{~h} \text { after } \\ \text { extubation }\end{array} & \begin{array}{c}\text { At ICU } \\ \text { discharge }\end{array} & \begin{array}{c}\text { At hospital } \\ \text { discharge }\end{array}\end{array}$

$\begin{array}{lll}\sqrt{ } & \sqrt{ } & \sqrt{ } \\ \sqrt{ } & \sqrt{ } & \sqrt{ } \\ \sqrt{ } & \sqrt{ } & \sqrt{ }\end{array}$

$\sqrt{ }$

$\sqrt{ }$

$\sqrt{ }$

$\sqrt{ }$

$\sqrt{ }$

ICU: intensive care unit; MIP: maximal inspiratory pressure; MEP: maximal expiratory pressure; MRC: Medical Research Council; SF-36: 36-item short form survey. 


\section{Discussion}

Patients that are difficult to wean off mechanical ventilation usually have a prolonged ICU stay that is associated with muscle atrophy and reduced muscle strength. Once these patients have survived a critical illness, they will then have to face significantly reduced functional ability and quality of life and will be in need of a long period of rehabilitation. In addition, these patients are at risk of a $6 \%$ to $50 \%$ hospital mortality rate [23]. Thus, the aim of this study is to improve the weaning process and as a consequence the patients' quality of life. Both IMT and HFNC have been used separately in critically ill patients with a positive effect on weaning from mechanical ventilation. IMT can improve muscle strength [6-8], while HFNC can improve endurance by reducing the work of breathing [24]. MARTIN et al. [8] showed a significant difference in weaning rate $(71 \%$ versus $47 \%)$ by using an inspiratory muscle rehabilitation programme. Interestingly, they have proposed that strengthening the inspiratory muscles theoretically could correct the feedback discrepancy between respiratory drive and lung/chest expansion and may result in a lower perception of breathing effort [8]. On the other hand, HFNC application immediately after extubation seems to significantly reduce re-intubation rates compared to conventional oxygen devices, and it is not inferior to noninvasive ventilation in high risk for extubation failure patients [14, 25]. HFNC seems to support the respiratory system after extubation effectively, and this effect might be attributed to high flow of the administered gas, which could meet the ventilator demand of the patient after extubation. If a combination of these novel techniques proves effective, this might significantly contribute to the treatment of patients who are difficult to wean and at high risk of re-intubation. In times of financial crisis, it is crucial to find and establish cost-effective procedures. It is well documented that reducing ICU length of stay and minimising its side-effects could have enormous economic benefit to our health systems worldwide [26].

Conflict of interest: None declared.

Support statement: All authors would like to acknowledge the support of the "Special Account for Research Grants" of the National and Kapodistrian University of Athens, Greece.

\section{References}

1 Boles JM, Bion J, Connors A, et al. Weaning from mechanical ventilation. Eur Respir J 2007; 29: 1033-1056.

2 Díaz MC, Ospina-Tascón GA, Salazar C BC. Respiratory muscle dysfunction: a multicausal entity in the critically ill patient undergoing mechanical ventilation. Arch Bronconeumol 2014; 50: 73-77.

3 Heunks LM, van der Hoeven JG. Clinical review: the ABC of weaning failure - a structured approach. Crit Care 2010; 14: 245.

4 Petrof BJ, Jaber S, Matecki S. Ventilator-induced diaphragmatic dysfunction. Curr Opin Crit Care 2010; 16: 19-25.

5 Gosselink R, Bott J, Johnson M, et al. Physiotherapy for adult patients with critical illness: recommendations of the European Respiratory Society and European Society of Intensive Care Medicine Task Force on Physiotherapy for Critically Ill Patients. Intensive Care Med 2008; 34: 1188-1199.

6 Funk GC, Anders S, Breyer MK, et al. Incidence and outcome of weaning from mechanical ventilation according to new categories. Eur Respir J 2010; 35: 88-94.

7 Patsaki I, Papadopoulos E, Sidiras G, et al. The effectiveness of inspiratory muscle training in critically ill patients from mechanical ventilation. Hospital Chronicles 2013; 8: 86-90.

8 Martin DA, Smith BK, Davenport PD, et al. Inspiratory muscle strength training improves weaning outcome in failure to wean patients: a randomised trial. Crit Care 2011; 15: R84.

9 Bissett BM, Wang J, Neeman T, et al. Which ICU patients benefit most from inspiratory muscle training? Retrospective analysis of a randomized trial. Physiother Theory Pract 2019; in press [https://doi.org/10.1080/ 09593985.2019.1571144].

10 Vorona S, Sabatini U, Al-Maqbali S, et al. Inspiratory muscle rehabilitation in critically ill adults: a systematic review and meta-analysis. Ann Am Thorac Soc 2018; 15: 735-744.

11 O'Driscoll BR, Howard LS, Davison AG. BTS guideline for emergency oxygen use in adult patients. Thorax 2008; 63: Suppl. VI, vil-vi68.

12 Hernández G, Roca $\mathrm{O}$, Colinas L. High-flow nasal cannula support therapy: new insights and improving performance. Crit Care 2017; 21: 62.

13 Ischaki E, Pantazopoulos I, Zakynthinos S. Nasal high flow therapy: a novel treatment rather than a more expensive oxygen device. Eur Respir Rev 2017; 26: 170028.

14 Maggiore SM, Idone FA, Vaschetto R, et al. Nasal high-flow versus Venturi mask oxygen therapy after extubation. Effects on oxygenation, comfort, and clinical outcome. Am J Respir Crit Care Med 2014; 190: 282-288.

15 Hernández G, Vaquero C, González P, et al. Effect of postextubation high-flow nasal cannula vs conventional oxygen therapy on reintubation in low-risk patients: a randomized clinical trial. JAMA 2016; 315: 1354-1361.

16 Hoffman M, Van Hollebeke M, Clercx B, et al. Can inspiratory muscle training improve weaning outcomes in difficult to wean patients? A protocol for a randomized trial (IMweanT study). BMJ Open 2018; 8: e021091.

17 Ritchie JE, Williams AB, Gerard C, et al. Evaluation of a humidified nasal high-flow oxygen system, using oxygraphy, capnography and measurement of upper airway pressures. Anaesth Intensive Care 2011; 39: $1103-1110$.

18 Tzanis G, Vasileiadis I, Zervakis D, et al. Maximum inspiratory pressure, a surrogate parameter for the assessment of ICU-acquired weakness. BMC Anesthesiol 2011; 11: 14.

19 Chang AT, Boots RJ, Brown MG, et al. Reduced inspiratory muscle endurance following successful weaning from prolonged mechanical ventilation. Chest 2005; 128: 553e9. 
Fan E, Ciesla ND, Truong AD, et al. Inter-rater reliability of manual muscle strength testing in ICU survivors and simulated patients. Intensive Care Med 2010; 36: 1038-1043.

21 Kidd D, Stewart G, Baldry J, et al. The functional independence measure: a comparative validity and reliability study. Disabil Rehabil 1995; 17: 10-14.

22 Chrispin PS, Scotton H, Rogers J, et al. Short Form 36 in the intensive care unit: assessment of acceptability, reliability and validity of the questionnaire. Anaesthesia $1997 ; 52$ : 15-23.

23 Mauri T, Turrini C, Eronia N, et al. Physiologic effects of high-flow nasal cannula in acute hypoxemic respiratory failure. Am J Respir Crit Care Med 2017; 195: 1207-1215.

24 Armando CG, Altamiro TP, Costa-Pereira A. Quality of life after intensive care - evaluation with EQ-5D questionnaire. Intensive Care Med 2002; 28: 898-907.

25 Hernandez G, Vaquero C, Colinas L, et al. Effect of post-extubation high-flow nasal cannula vs noninvasive ventilation on reintubation and post-extubation respiratory failure in high-risk patients: a randomized clinical trial JAMA 2016; 316: 1565-1574.

26 Ambrosino N, Gabbrielli L. The difficult to wean patient. Expert Rev Resp Med 2010; 4: 685-692. 